\title{
The application of the weak electric field pulse to measure the pseudo-Stark split by the photon echo beating
}

\author{
V.N.Lisin, A.M.Shegeda, V.V.Samartsev \\ Zavoisky Physical-Technical Institute, Russian Academy of Sciences, Kazan, \\ 420029 Russia \\ E-mail: vlisin@kfti.knc.ru
}

November 2014

\begin{abstract}
New scheme for determining the pseudo-Stark splitting of optical lines has been proposed and experimentally realized. A pulse of a weak electric field leads to occurrence of relative phase shifts of the excited dipoles and, as consequence, to beating of a photon echo wave form if electric pulse overlaps in time with echo-pulse. The value of the pseudo-stark splitting is the inverse period of the beats. The photon echo beating of the R1-line in Ruby have been observed. The pseudo-Stark splitting has been determined and it's value have been compared with the known literary data.
\end{abstract}

PACS numbers: $42.50 . \mathrm{Md}, 42.65 . \mathrm{Vh}$

Keywords: photon echo, phase control, low electric field, pseudo-Stark splitting, $\mathrm{Cr}^{3+}$, Ruby 


\section{Introduction}

Paper purpose is to observe the the photon echo beating of the R1-line in Ruby, the pulse applying weak electric field, and measure the pseudo-Stark splitting.

Photon echo is coherent radiation of medium in form of short pulse, caused by restoration of phase of separate radiators after the change of a sign on relative frequency of radiators. If perturbation splits optical line, for example, on two lines, this means that the frequency of transitions in two groups of radiators are shifted by different values. If a pulse of the perturbation overlaps in time with echo-pulse then the echo waveform changes and photon echo beats must be. The photon echo beats have been observed firstly in systems in which the Zeeman splitting is realised [1, 2, 3. In systems, in which the pseudo-Stark effect is manifested, the line splitting in electric field are due to the different Stark shifts at different lattice sites (as you cay see from figure 1). It is well known that in Ruby there are two kinds of chromium sites, A and B [4. Transformation of the A site into the B site is only possible by symmetry operations involving inversion around the chromium ion, while transformation of $\mathrm{A}$ to A or B to B is achieved solely by translation or by both translation and rotation around the optic axis. The $\mathrm{A}$ and $\mathrm{B}$ sites are energetically equivalent in the absence of an electric field. The shifts $\delta \nu$ of transition frequencies of $\mathrm{A}$ and $\mathrm{B}$ ions in the electric field equal in magnitude but differ in sign and the optical line splits:

$$
\begin{aligned}
& \delta \nu= \pm Z / 2, \\
& Z=2 \partial \nu / \partial E \cdot E,
\end{aligned}
$$

Here $Z$ is optical line splitting, $E$ is the component of the external electric field along the crystal axis $C_{3}$ in Ruby, the parameter $\partial \nu / \partial E$ is proportional to the difference of the values of diagonal matrix elements of the operator of the electric dipole moment in the ground and excited states of the R1 line. Relative phase $\varphi$ of these dipole groups is not equal to zero as result:

$\varphi(t)=2 \pi \int_{t_{0}}^{t} Z\left(t^{\prime}\right) d t^{\prime}$.

Echo intensity oscillates versus phase [1, 2, 3] :

$I(t)=I_{0}\langle\cos (\varphi(t) / 2)\rangle^{2}$,

where $I_{0}(t)$ is intensity of an echo when a electric pulse (EP) off, $t_{0}$ is time of the beginning of action of a EP, $Z$ is pseudo-Stark splitting of optical line (twice the value of the pseudo-stark shift), $\langle\ldots\rangle$ denotes averaging by optically excited volume.

Let's pass to consideration of experimental technics.

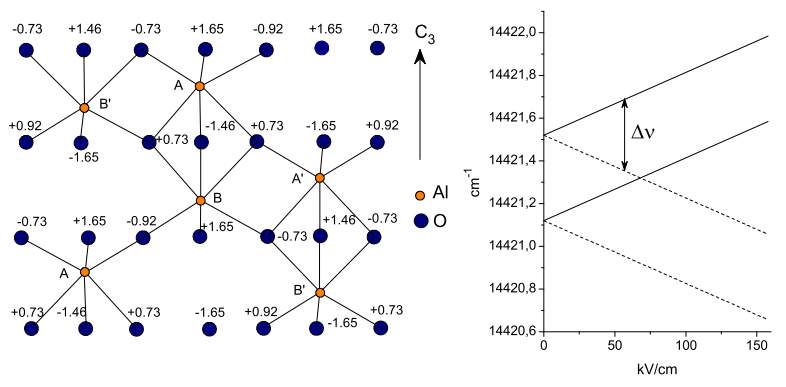

Figure 1. At the left Ruby structure 4] is shown. At the right the splitting of a R1-line of absorption of a Ruby is conditionally shown in external constant electric field in size to $160 \mathrm{kV} / \mathrm{cm}$ [5]. Continuous and dashed lines belong to ions of chrome of different type (A and B ions).

\section{Experimental conditions}

We observed the photon echo in the backward mode on the R1-line of the $\left(\mathrm{Al}_{2} \mathrm{O}_{3}: \mathrm{Cr}^{3+}\right)$ at temperature of $2 \mathrm{~K}$. The laser pulses with duration of $13 \mathrm{~ns}$ have been generated by an oxazine- 1 dye laser. The spectral FWHM is about $6 \mathrm{GHz}$. Delay time between the laser pulses is less than or equal to $64 \mathrm{~ns}$. Polarization of the laser pulses is linear. The photon echo signals were detected on a photomultiplier and fed to a Tektronix TDS 2022 digital oscilloscope. All experimental measurements were controlled with LabVIEW software. The geometry of the experiment is shown in figure 2. The electric field is obtained by applying a voltage difference to a pair of electrodes press down to an opposite faces of the crystal with the resulting electric field parallel to $C_{3}$-axis. Each electrode is thin copper plate with hole in center. Hole diameter is $1 \mathrm{~mm}$. Axis $\mathrm{X}$ passes through the centers of the holes of the electrodes, directed in parallel to the crystal $C_{3}$-axis. The origin is in the center between the plates: $x=0$. The direction of propagation of the laser beams are approximately parallel to the $C_{3}$-axis. The angle between the rays of the first and second laser pulses is about 4,5 degrees. The diameter of the focused beams in sample is $0.15 \mathrm{~mm}$. Dependence of the intersection area of beams versus $x$ is shown in figure 2 .

Voltage pulses formed by a generator with avalanche transistors are applied to the electrodes synchronously with the laser pulses. The half max voltage pulse duration $\tau_{U}$ equal $34 \mathrm{~ns}$ and upper flat part duration is $23 \mathrm{~ns}$ as you can see in figure 2 . The maximum amplitude of a voltage difference is equal $268 \mathrm{~V}$. The voltage amplitude was varied with a step of $1 \mathrm{~dB}$.

The electric field between the electrodes with holes was calculated by numerical solution of the Poisson equation. We Take into account that the electrodes are 

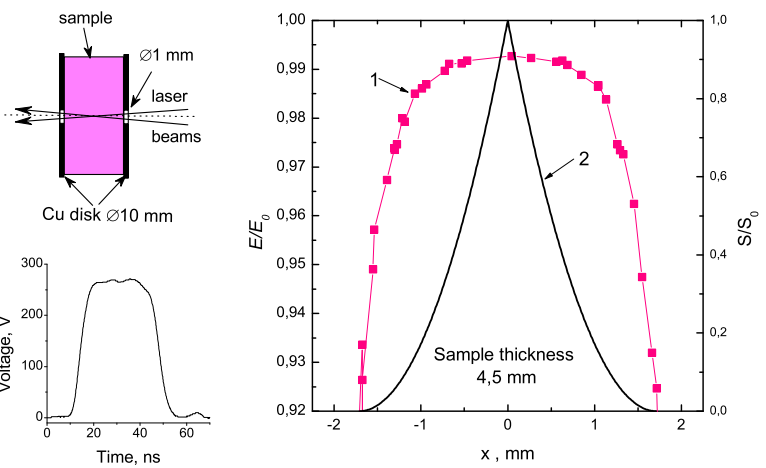

Figure 2. At the left above the experiment geometry is resulted. Arrows represent the rays of the first and second laser pulses, the dot line depicts the $C_{3}$ and $X$-axes. The condenser is formed by two copper disks in diameter of $10 \mathrm{~mm}$ with holes in the centre in diameter of $1 \mathrm{~mm}$. Disks are densely pressed to the sample. The first and second laser pulses in diameter $0.15 \mathrm{~mm}$ transit in holes of disks at an angle 4,5 degrees rather each other. At the left below you can see a waveform of a pulse of the voltage difference submitted on condenser. The calculated values of the electric field $E / E_{0}$ (line 1 ) and the area of beams intersection $S / S_{0}$ (line 2) versus $x$ are shown at the right. The origin is in the center between the plates: $\mathrm{x}=0 . E_{0}$ is defined in (4), and $S_{0}$ is the cross-sectional area of the laser beam $S_{0}=\pi(0.15 / 2)^{2}$ $\mathrm{mm}^{2}$.

equipotential surfaces and electric field on the surface of the electrodes directed normal to the electrode surfaces.

Calculations showed (figure 2) that the value of the component of the electric field parallel to the $C_{3}$ axis in the center of the optically excited volume is equal 0,993 to an infinite flat field of the capacitor without holes $E_{0}$ :

$E_{0}=U / d$,

where $U$ is voltage difference between the electrodes and $d$ is the sample thickness.

From the calculations it follows that with good accuracy the relation is valid

$\langle\cos (\varphi(t) / 2)\rangle=\cos (\langle\varphi(t) / 2\rangle)$

in determining the times at which the intensity (3) minima are achieved. We take into consideration dependence of the area of intersection of beams on $\mathrm{x}$ during the averaging over the optically excited volume. See line 2 in figure 2 at the right. The average value of the electric field is

$\langle E\rangle=0.987 U / d=0.987 E_{0}$

Given the fact that the phase change for the period is equal to $2 \pi$, make the approximation

$$
\langle\varphi(t+T)\rangle-\langle\varphi(t)\rangle=2 \pi=2 \pi\langle Z\rangle T,
$$

which is true only on a flat part of the electric pulse form. From (7) you can express pseudo-stark frequency through the reverse period modulation

$$
\langle Z\rangle=1 / T=2 \partial \nu / \partial E \cdot\langle E\rangle
$$

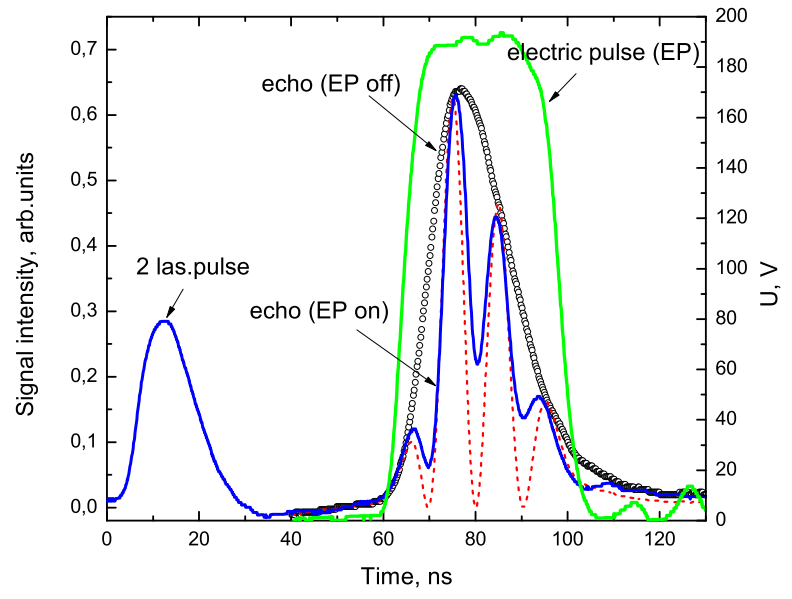

Figure 3. Waveforms of electric pulse (EP) and photon echo when the EP on and off in Ruby with ${ }^{52} \mathrm{Cr}$. Dashed line simulation of intensity of a photon echo according to expressions (3) 20- 9)

and the difference dipole moments using experimentally measured value

$2 \frac{\partial \nu}{\partial E}=\frac{\partial\langle Z\rangle}{\partial\langle E\rangle}=\frac{\partial(1 / T)}{\partial\langle E\rangle}=\frac{d}{0.987} \frac{\partial(1 / T)}{\partial U}$.

\section{Results of experiment}

In figure 3 waveforms of observable R1-line echo signals in Ruby samples. You can see also EP waveforms $U$.

From the waveforms of echo signals observed at various times of the beginning of action of the EP, similar to those shown in figure 3 , time intervals between the nearest minima in the echo signals were measured and the average value $T$ of the period has been determined. Also the electric field amplitudes $E_{0}$ and $\langle E\rangle$ in the region of the top flat time area of voltage pulse has been calculated using equations (4) and (6). The dependence of the inverse average period $1 / T$ versus the $\langle E\rangle$ has been built. Figure 4 shows these dependencies for Ruby samples of different thickness and are enriched for different isotopes of chromium ion ${ }^{52} \mathrm{Cr}$ and ${ }^{53} \mathrm{Cr}$. We take into account that echo modulation is absent, i.e. $1 / T=0$ when $\langle E\rangle=0$.

From figure 4 the values of following parameters are defined:

$$
\begin{aligned}
& { }^{52} \mathrm{Cr}, d=4.5 \mathrm{~mm} \\
& \partial(1 / T) / \partial\langle E\rangle=0.214\left(M H z V^{-1} \mathrm{~cm}\right), \\
& { }^{53} \mathrm{Cr}, d=4.2 \mathrm{~mm} \\
& \partial(1 / T) / \partial\langle E\rangle=0.214\left(M H z V^{-1} \mathrm{~cm}\right) .
\end{aligned}
$$

Taking into account (10) we have

$\left.2 \partial \nu / \partial E=0.214\left(M H z V^{-1} \mathrm{~cm}\right)\right\}^{52} \mathrm{Cr},{ }^{53} \mathrm{Cr}$. 


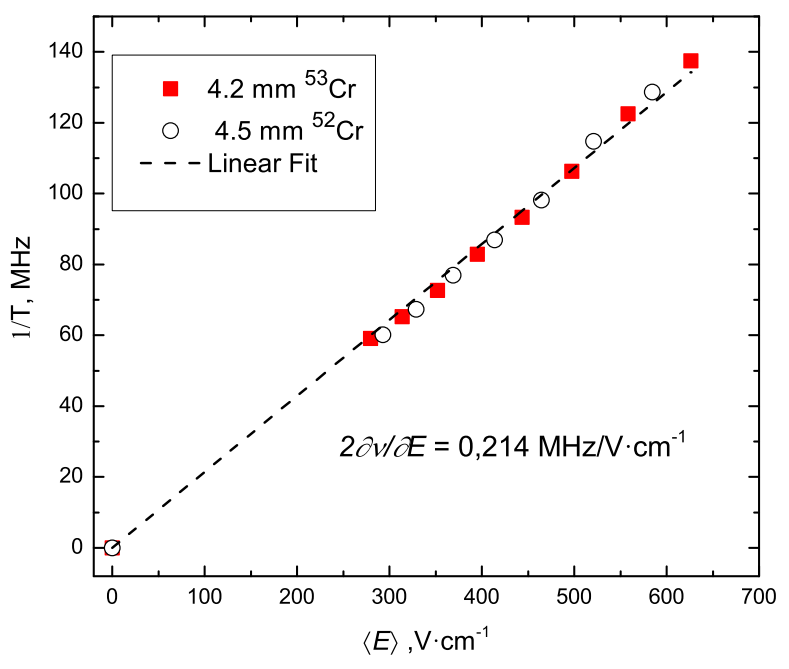

Figure 4. Inverse average modulation period of the echo waveform versus $\langle E\rangle$. The $\langle E\rangle$ is average value of the electric field over the optically excited volume (6), where $U$ is amplitude of a voltage pulse for the time in the region of a top flat of the voltage pulse.

\section{Discussion of results}

The obtained values of the pseudo-stark splitting parameter are in good agreement with known literature data. For comparison, we give the values obtained by other methods in the same units as in (11):

$2 \partial \nu / \partial E=0.176[5], 0.228[\underline{6}], 0.220 \pm 0.016[7$ ]

The main reason for the error in the determination of the pseudo-stark splitting parameter is unstable laser pulse shape and, consequently, unstable waveform of the photon echo. In the ideal case, when the initial shape of the echo signal and the shape of the EP close to the rectangular, it is enough a single waveform echo when the EP on to determine the modulation period $T$. Enough to know the amplitude of the EP and the time between the nearest minima in the resulting echo response. If the shape of the echo signal is completely arbitrary, but repetitive from pulse to pulse, knowing the EP dependence versus time and time $t_{0}$ when the voltage pulse on, it is easy to calculate for a given $\partial \nu / \partial E$ the waveform of the resulting echo response . Comparing the period of the modulation of the echo signal with the experiment, we can find the $\partial \nu / \partial E$. In this case, you have two echo waveforms: with EP off and EP on. If the shape of the echo signal is completely arbitrary and does not repetitive from pulse to pulse, it is impossible to know exactly the shape of the echo when EP off. In this case, to reduce the error we must submit the EP that creates in the echo waveform as much as possible minima and then to calculate the average the time between the nearest minima. Preliminary results were presented at conferences IWQO-2015 [8].

\section{Conclusions}

By controlling the relative phase of the excited dipoles with weak perturbations can significantly change the resultant dipole moment of the system. This can occur in cases where the coherence is important. In our study we measured the pseudo-stark splitting of optical line and determined electric parameter $\partial \nu / \partial E$ of the paramagnetic ion. If the electric parameters are known, it is possible to determine the amplitude of the EP, using the relation (8). For example, if the electric field is created by the pulse changes the dipole-dipole interaction of the ion with the environment. This can be used to determine the distance to the centers of the environment by measuring the waveform of the echo modulation periods.

\section{Acknowledgments}

This work was supported by the RAS program Fundamental optical spectroscopy and its applications and by the RFBR grants no. 14-02-00041a.

\section{References}

[1] Lisin V, Shegeda M 2012 Modulation of the Shape of the Photon Echo Pulse by a Pulsed Magnetic Field: Zeeman splitting in LiLuF4:Er3 + and LiYF4:Er3 + JETP Letters $96328-332$

[2] Lisin V, Shegeda M, Samartsev V 2015 The application of the weak magnetic field pulse to measure g-factors of ground and excited optical states by a photon echo method Laser Phys. Lett. 12025701

[3] Lisin V, Shegeda M, Samartsev V 2015 New possibilities of photon echo: determination of ground and excited states g-factors applying a weak magnetic field pulse Journal of Physics: Conference Series 613012013

[4] Wyckoff R 1961 Crystal Structures (Interscience Publishers, New York, )

[5] Kaiser W 1961 SPLITTING OF THE EMISSION LINES OF RUBY BY AN EXTERNAL ELECTRIC FIELD PHYS. Rev.Lett. 6605

[6] COHEN M AND BLOEMBERGEN N. 1964 Magnetic- and Electric-Field Effects of the B1and B2 Absorption Lines in Ruby PHYS. REV. 135 A950

[7] Szabo A and Kroll M 1978 Stark-induced optical transients in Ruby OPTICS LETTERS 2 10-12

[8] Lisin V, Shegeda M, Samartsev V 2015 Definition of shifts of optical transitions frequencies due to pulse perturbation action by the photon echo signal form EPJ Web of Conferences 10307004 